\title{
Generalized Equilibrium Problems Related to Ky Fan Inequalities
}

\author{
Ionel Rovenţa \\ Department of Mathematics, University of Craiova, A. I. Cuza Street, No. 13, 200585 Craiova, Romania \\ Correspondence should be addressed to Ionel Rovenţa; ionelroventa@yahoo.com
}

Received 29 October 2013; Accepted 1 January 2014; Published 12 February 2014

Academic Editor: Chong Li

Copyright (C) 2014 Ionel Rovenţa. This is an open access article distributed under the Creative Commons Attribution License, which permits unrestricted use, distribution, and reproduction in any medium, provided the original work is properly cited.

We study a generalized equilibrium problem by using a nonsymmetric extension of Ky Fan's inequality. As an application, we present a fixed point type algorithm inspired by a model from Tada and Takahashi (2007).

\section{Introduction}

In this paper, we study a generalized equilibrium problem which is connected with some results from $[1,2]$. More precisely, by using a nonsymmetric extension of Ky Fan's inequality, we solve a special class of equilibrium problems, which can be fitted into the field of minimax inequalities. By using such type of generalized equilibrium problems we consider a fixed point type algorithm based on two iterative sequences. Such type of problems have been extensively studied [1-4]. Other interesting results concerning equilibrium problems can be found in $[5,6]$.

Several methods for solving systems of equilibrium problems in Hilbert spaces are studied in [3]. The proposed methods include proximal-like block-iterative algorithms for general systems, as well as regularization and splitting algorithms for single equilibrium problems.

On the other hand, the study of such inequalities, fixed point of nonlinear mappings, and their approximation algorithms can be successfully used in order to solve some equilibrium problems. In [1] two iterative algorithms for a generalized Ky Fan inequality and a fixed point problem of asymptotically strict pseudocontractions are studied. A strong convergence theorem is also obtained based on the hybrid projection method in Hilbert spaces. Moreover, in $[2,7,8]$, weak and strong convergence theorems of finding a common element of the set of fixed points of a nonexpansive mapping and the set of solutions of the equilibrium problem have been studied.
Let us consider a function $f: C \times C \rightarrow \mathbb{R}$, where $C$ is a nonempty, compact, and convex subset of a Hilbert space $\mathscr{H}$.

The equilibrium problem, denoted by (EP), consists in finding an element $\bar{x} \in C$ such that

$$
f(\bar{x}, y) \geq 0 \quad(y \in C) .
$$

The function $f$ is called monotone if

$$
f(x, y)+f(y, x) \leq 0 \quad(x, y \in C) .
$$

Let $g: C \rightarrow C$ be one to one continuous functions, $F$ : $C \times C \rightarrow \mathbb{R}$ and $r>0$

Let $x \in \mathscr{H}$. The generalized equilibrium problem, denoted by (GEP), consists in finding an element $z \in C$ such that

$$
F(z, y)+\frac{1}{r}\left\langle y-g^{-1}(z), g^{-1}(z)-x\right\rangle \geq 0 \quad(y \in C) .
$$

We remark that if we choose $x=g^{-1}(z)$ we obtain the classical equilibrium problem (EP). The aim of the paper is to solve the generalized equilibrium problem (3) under the suitable conditions imposed to the function $F$ and to study some related problems.

Note that many classes of problems can be fitted in the class of equilibrium problems. In the following, we present such classes of problems which can be solved as an equilibrium problem. 
First example consists of an optimization problem. Let $T$ : $C \rightarrow \mathbb{R}$ be a given mapping. We are looking for an element $\bar{x} \in C$ such that

$$
T(\bar{x}) \leq T(y) \quad(y \in C) .
$$

We remark that $\bar{x}$ solves the minimization problem (4) if and only if it solves the equilibrium problem (EP), where $f(x, y)=T(y)-T(x)$. Note that in this case we have

$$
f(x, y)+f(y, x)=0 ;
$$

thus, $f$ is monotone.

On the other hand, if $T$ is a convex differentiable mapping, then the optimization problem (4) is equivalent with the existence of an element $\bar{x} \in C$ such that the following variational inequality holds:

$$
\langle\nabla T(\bar{x}), y-\bar{x}\rangle \geq 0 \quad(y \in C) .
$$

Nevertheless, if we consider $f(x, y)=\langle\nabla T(x), y-x\rangle$, for all $x, y \in C$, the variational inequality (6) and the equilibrium problem (EP) are equivalent.

Moreover, since $T$ is convex and differentiable, we have that

$$
f(x, y)+f(y, x)=-\langle\nabla T(x)-\nabla T(y), x-y\rangle \leq 0 ;
$$

hence, $f$ is monotone.

Moreover, we can consider a generalized version of (6) given by the existence of an element $\bar{x} \in C$ such that

$$
\langle T(\bar{x}), y-\bar{x}\rangle \geq 0 \quad(y \in C) .
$$

If we consider $f(x, y)=\langle T x, y-x\rangle$, we have the equivalence between (8) and equilibrium problem (EP).

The saddle point problems can be viewed also in the context of equilibrium problems. If $\phi: C_{1} \times C_{2} \rightarrow \mathbb{R}$, then $\left(\bar{x}_{1}, \bar{x}_{2}\right) \in C_{1} \times C_{2}$ is a saddle point if and only if

$$
\phi\left(\bar{x}_{1}, y_{2}\right) \leq \phi\left(y_{1}, \bar{x}_{2}\right) \quad\left(\left(y_{1}, y_{2}\right) \in C_{1} \times C_{2}\right) .
$$

The classical definition of a saddle point can be obtained by taking in (9) $y_{1}=\bar{x}_{1}$, respectively $y_{2}=\bar{x}_{2}$, which gives

$$
\phi\left(\bar{x}_{1}, y_{2}\right) \leq \phi\left(\bar{x}_{1}, \bar{x}_{2}\right) \leq \phi\left(y_{1}, \bar{x}_{2}\right) \quad\left(\left(y_{1}, y_{2}\right) \in C_{1} \times C_{2}\right) .
$$

In order to formulate the saddle point problem as an equivalent equilibrium problem, we define $C=C_{1} \times$ $C_{2}$ and $f\left(\left(x_{1}, x_{2}\right),\left(y_{1}, y_{2}\right)\right)=\phi\left(y_{1}, x_{2}\right)-\phi\left(x_{1}, y_{2}\right)$. The function $f$ is also monotone, that is, $f\left(\left(x_{1}, x_{2}\right),\left(y_{1}, y_{2}\right)\right)+$ $f\left(\left(y_{1}, y_{2}\right),\left(x_{1}, x_{2}\right)\right)=0$.

We present now an application to economics, given by a Nash equilibrium problem. Suppose that we have a finite set $\mathscr{I}$ of $N$ players. Let $C_{i}$ be the finite set of pure strategies of player $i \in I$. We define $C=\prod_{i \in I} C_{i}$. For all $i \in I$, let $f_{i}$ : $C_{i} \rightarrow \mathbb{R}$ be the loss function of player $i$. For arbitrary $x=$ $\left(x_{1}, \ldots, x_{n}\right) \in C$, we define $\bar{x}_{i}=\left(x_{1}, \ldots, x_{i-1}, x_{i+1}, \ldots, x_{n}\right)$.

An N-tuple $\bar{x} \in C$ is a Nash equilibrium point if for all $i \in I$ we have that

$$
f_{i}(\bar{x}) \leq f_{i}\left(\bar{x}_{i}, y_{i}\right) \quad\left(y_{i} \in C_{i}\right)
$$

It can be shown that $\bar{x}$ is a Nash equilibrium point, more precisely a solution of (11), if and only if it is a solution of the equilibrium problem (EP), where $f: C \times C \rightarrow \mathbb{R}$ is given by $f(x, y)=\sum_{i \in I} f_{i}\left(\bar{x}_{i}, y_{i}\right)-f_{i}(x)$.

Let us consider now a fixed point problem. If $T: C \rightarrow C$ is a map, then $\bar{x} \in C$ is a fixed point of $T$ if and only if $\bar{x}$ is a solution of the equilibrium problem (EP), where $f(x, y)=$ $\langle x-T x, y-x\rangle$.

Note that the function $f(x, y)=\langle x-T x, y-x\rangle$ is monotone if and only if

$$
\langle T x-T y, x-y\rangle \leq\|x-y\|^{2}, \quad(x, y \in C),
$$

which says that $f$ is monotone if and only if it is nonexpansive.

The rest of the paper is organized as follows. In Section 2, we use some minimax theory elements in order to obtain a nonsymmetric form of Ky Fan's inequality for the case of quasiconvex functions. In Section 3, we solve the generalized equilibrium problem (GEP) by using the extension of Ky Fan inequality and we discuss some related problems and applications.

\section{A Nonsymmetric Extension of Ky Fan's Inequality}

In this section, we present a nonsymmetric extension of $\mathrm{Ky}$ Fan's inequality for the case of quasi-convex functions. In a similar way, Ky Fan's inequality has been extended in the context of metric spaces with global nonpositive curvature. More details and results on this topic can be found in [9-11].

Suppose that $C$ is a nonempty compact and convex subset of a linear topological space E. Recall the fact that Ky Fan's minimax inequality asserts that any function $f: C \times C \rightarrow \mathbb{R}$ which is upper semicontinuous in the first variable and quasiconvex in the second variable verifies the following minimax inequality:

$$
\max _{x \in C} \inf _{y \in C} f(x, y) \geq \inf _{z \in C} f(z, z)
$$

Notice that Ky Fan's inequality is equivalent to Brouwer's fixed point theorem. See [12, page 212].

Definition 1 . We say that a function $g: E \rightarrow F$ is quasiconvex if

$$
\begin{array}{r}
g((1-\lambda) x+\lambda y) \leq \max \{g(x), g(y)\} \\
(x, y \in E, \lambda \in(0,1)) .
\end{array}
$$

Firstly, we present the classical Knaster-KuratowskiMazurkiewicz (KKM) theorem, which will be used later.

Theorem 2 (KKM). Assume that for every point $x$ belonging to a nonempty set $X \subset E$ there exists a closed subset $M(x) \subset X$. Suppose that the following property:

$$
\operatorname{conv} F \subset \bigcup_{x \in F} M(x)
$$


holds for every finite set $F \subset X$. Then for any finite subset $F \subset X$ one has

$$
\bigcap_{x \in F} M(x) \neq \emptyset
$$

Thus, if a subset $M(x)$ is compact, one has

$$
\bigcap_{x \in X} M(x) \neq \emptyset \text {. }
$$

We prove now a nonsymmetric extension of Ky Fan's inequality by using a continuous and onto function.

Theorem 3. Let D, F be two nonempty, compact, and convex subsets of $E$ and let $g$ be a continuous onto function $g: F \rightarrow$ $D$. Let $f: D \times F \rightarrow \mathbb{R}$ be a function which is upper semicontinuous in the first variable and quasiconvex in the second variable. Then, one has

$$
\max _{x \in D} \inf _{y \in F} f(x, y) \geq \inf _{z \in F} f(g(z), z) .
$$

Notice that Theorem 3 extends the classical Ky Fan inequality. More exactly, taking $D=F$ and $g(z)=z$ for all $z \in F$ in Theorem 3 we obtain (13).

Proof. Consider the family of sets

$$
\begin{array}{r}
M(g(y))=\left\{x \in D \mid f(x, y) \geq \inf _{z \in F} f(g(z), z)\right\} \\
(y \in F) .
\end{array}
$$

We will show that we can apply the results from KKM's theorem. First of all, we remark that $g(y) \in M(g(y)) \subset D$, for $y \in F$.

Let $A=\left\{g\left(y_{1}\right), g\left(y_{2}\right)\right\}$ be a subset of $D$. For more elements of $D$ the proof is similar. We need to show that

$$
\operatorname{conv} A \subset \bigcup_{i \in\{1,2\}} M\left(g\left(y_{i}\right)\right)
$$

that is,

$$
\begin{array}{r}
(1-\lambda) g\left(y_{1}\right)+\lambda g\left(y_{2}\right) \in M\left(g\left(y_{1}\right)\right) \cup M\left(g\left(y_{2}\right)\right) \\
(\lambda \in(0,1)) .
\end{array}
$$

Indeed, if the contrary is true, then for a $\lambda \in(0,1)$ we have

$$
\begin{aligned}
& f\left((1-\lambda) g\left(y_{1}\right)+\lambda g\left(y_{2}\right), y_{1}\right)<\inf _{z \in F} f(g(z), z), \\
& f\left((1-\lambda) g\left(y_{1}\right)+\lambda g\left(y_{2}\right), y_{2}\right)<\inf _{z \in F} f(g(z), z) .
\end{aligned}
$$

From the continuity of $g$, we infer the existence of an element $y_{\lambda} \in D$ such that

$$
(1-\lambda) g\left(y_{1}\right)+\lambda g\left(y_{2}\right)=g\left(y_{\lambda}\right),
$$

where $y_{\lambda}=\alpha_{0} y_{1}+\left(1-\alpha_{0}\right) y_{2}$ for an $\alpha \in(0,1)$.

Let $h:[0,1] \rightarrow F$ be a continuous function such that

$h(\alpha)=(1-\lambda) g\left(y_{1}\right)+\lambda g\left(y_{2}\right)-g\left(\alpha y_{1}+(1-\alpha) y_{2}\right)$.
It follows that

$$
\begin{gathered}
h(0)=(1-\lambda)\left(g\left(y_{1}\right)-g\left(y_{2}\right)\right), \\
h(1)=\lambda\left(g\left(y_{2}\right)-g\left(y_{1}\right)\right) .
\end{gathered}
$$

Hence, $h(0)$ and $h(1)$ are of contrary signs and we deduce the existence of an $\alpha_{0} \in[0,1]$ such that $h\left(\alpha_{0}\right)=0$.

We have

$$
\begin{gathered}
f\left(g\left(y_{\lambda}\right), y_{\lambda}\right) \\
=f\left((1-\lambda) g\left(y_{1}\right)+\lambda g\left(y_{2}\right), \alpha_{0} y_{1}+\left(1-\alpha_{0}\right) y_{2}\right) \\
\leq \max \left\{f\left((1-\lambda) g\left(y_{1}\right)+\lambda g\left(y_{2}\right), y_{1}\right),\right. \\
\left.\quad f\left((1-\lambda) g\left(x_{1}\right)+\lambda g\left(x_{2}\right), y_{2}\right)\right\} \\
<\inf _{z \in F} f(g(z), z) .
\end{gathered}
$$

Thus, we obtain a contradiction. Hence, we can apply KKM's theorem and we have that $\cap_{y \in F} M(g(y)) \neq \emptyset$. It follows that there exists $x_{0} \in D$ such that for every $y \in F$ we have

$$
\begin{gathered}
f\left(x_{0}, y\right) \geq \inf _{z \in F} f(g(z), z), \\
\inf _{y \in F} f\left(x_{0}, y\right) \geq \inf _{z \in F} f(g(z), z), \\
\operatorname{maxinf}_{x \in D} f(x, y) \geq \inf _{z \in F} f(g(z), z) .
\end{gathered}
$$

With similar arguments, we can prove the following theorem in the case of quasiconcavity.

Theorem 4. Let $D, F$ be two nonempty, compact, and convex subsets of $E$ and let $g: D \rightarrow F$ be a continuous onto function. Let $f: D \times F \rightarrow \mathbb{R}$ be a quasi-concave function in the first variable and lower semicontinuous in the second variable. Then, one has

$$
\min _{y \in F} \sup _{x \in D} f(x, y) \leq \sup _{z \in D} f(z, g(z))
$$

An important application of the above theorems is the existence of an $g$-equilibrium which generalizes the existence of the well-known concept of Nash equilibrium. See [10].

Theorem 5. Let $C=C_{1} \times C_{2} \times \cdots \times C_{n}$, where $C_{i}, i=1, \ldots, n$ are nonempty, compact, and convex subsets of $E$, let $g=$ $\left(g_{1}, g_{2}, \ldots, g_{n}\right): C \rightarrow C$ be a continuous onto function, and let $f_{i}: C \rightarrow C$ be a function which is lower semicontinuous in the second variable and $x_{i} \rightarrow\left(f_{i}\right)\left(y_{1}, \ldots, g\left(x_{i}\right), \ldots, y_{n}\right)$ is quasiconcave for every $i=1, \ldots, n$. Then, there exists an $y \in C$ such that

$$
f_{i}(y) \leq f_{i}\left(y_{1}, \ldots, g\left(x_{i}\right), \ldots, y_{n}\right)
$$

for every $x_{i} \in C_{i}, i=1, \ldots, n$. 


\section{Main Results}

In this section, we use the above nonsymmetric extension of Ky Fan's inequality in order to solve the generalized equilibrium problem (GEP). In the following, we present the strategy of solving the generalized equilibrium problem (GEP) which will be used later to present a fixed point type algorithm.

Let $\mathscr{H}$ be a Hilbert space, $x \in \mathscr{H}$, and $r>0$. Recall here the generalized equilibrium problem, which consists in finding an element $z \in C$ such that

$$
F(z, y)+\frac{1}{r}\left\langle y-g^{-1}(z), g^{-1}(z)-x\right\rangle \geq 0 \quad(y \in C),
$$

where $F: C \times C \rightarrow \mathbb{R}$ is a suitable function and $g: C \rightarrow C$ is a one to one function.

We pass now to the problem of proving the existence of an element $z \in C$ which solves (30).

Lemma 6. Let $g: C \rightarrow C$ be a continuous bijective function and let $F: C \times C \rightarrow \mathbb{R}$ be a quasiconvex function in the second variable and upper semicontinuous in the first variable which verifies

$$
F(g(z), z) \geq 0 \quad(z \in C) .
$$

Then there exists $z \in C$ a solution of problem (30).

Remark 7. Here, the novelty consists of the fact that we can solve (30) by imposing weaker conditions than those from [1$3]$. More precisely, the symmetric condition $f(z, z)=0$ is replaced with a nonsymmetric one, given by $F(z, g(z)) \geq 0$, for each $z \in C$.

Proof. Let us consider $F_{g}(z, y)=F(z, y)+(1 / r)(y-$ $\left.g^{-1}(z), g^{-1}(z)-x\right)$. Since the function $F$ is quasiconvex in the second variable (as the sum of a quasi-convex function and a linear function) and upper semicontinuous in the first variable, then by applying Theorem 3 we obtain

$$
\sup _{z \in C} \inf _{y \in C} F_{g}(z, y) \geq \inf _{z \in C} F_{g}(g(z), z) \text {. }
$$

In fact, (32) says the fact that there exists $z \in C$ such that

$$
F_{g}(z, y) \geq \inf _{z \in C} F_{g}(g(z), z)=\inf _{z \in C} F(g(z), z) \geq 0 \quad(y \in C) .
$$

Hence, we have an element $z \in C$ which solves (30) and the proof is finished.

Note that Lemma 6 will be used to study two iterative sequences which converge to a common element of the set attached to a nonexpansive mapping and the set of solutions of an equilibrium problem in a Hilbert space. The ideas are based on several results from [2], when the conditions imposed are symmetric $f(z, z)=0$. By assuming weaker conditions we prove that similar results hold.

Definition 8. Let $C$ be a nonempty, closed, and convex subset of a real Hilbert space $\mathscr{H}$ and let $g: C \rightarrow C$ be a continuous and bijective function. We say that a bifunction $F: C \times C \rightarrow$ $\mathbb{R}$ verifies the $g$-equilibrium conditions if

(a) $F(g(x), x)=0$, for all $x \in C$;

(b) $F(g(x), y)+F(g(y), x) \leq 0$, for all $x, y \in C$;

(c) $\lim \sup _{t \downarrow 0} F(t z+(1-t) x, y) \leq f(x, y)$, for all $x, y, z \in$ C;

(d) $F(x, \cdot)$ is convex and lower semicontinuous for each $x \in C$.

Definition 9. The generalized resolvent of a bifunction $F: C \times C \rightarrow \mathbb{R}$ is the set-valued operator $J_{F}: \mathscr{H} \rightarrow 2^{C}$ defined by

$$
\begin{gathered}
J_{F}(x)=\left\{z \in C \mid F(z, y)+\left\langle y-g^{-1}(z),\right.\right. \\
\left.\left.g^{-1}(z)-x\right\rangle \geq 0, \forall y \in C\right\} .
\end{gathered}
$$

Since $g$ is an onto function we have that

$$
\begin{aligned}
& J_{F}(x) \\
& =\left\{z \in C \mid F\left(z, g^{-1}(y)\right)+\left\langle g^{-1}(y)-g^{-1}(z),\right.\right. \\
& \left.\left.\qquad g^{-1}(z)-x\right\rangle \geq 0, \forall y \in C\right\} .
\end{aligned}
$$

Lemma 10. Suppose that $F: C \times C \rightarrow \mathbb{R}$ satisfies the $g$ equilibrium conditions and let

$$
S_{F}=\{x \in C \mid F(x, y) \geq 0, \forall y \in C\} .
$$

Then, one has

(i) $\operatorname{dom} J_{F}=\mathscr{H}$;

(ii) $J_{F}$ is single-valued and firmly nonexpansive; that is, for any $x, y \in \mathscr{H}$

$$
\left\|J_{F}(x)-J_{F}(y)\right\|^{2} \leq\left\langle J_{F}(x)-J_{F}(y), x-y\right\rangle ;
$$

(iii) for each $x \in C g(x) \in J_{F}(x)$ is equivalent with the fact that $g(x) \in S_{F}$;

(iv) $S_{F}$ is closed and convex.

Proof. (i) By using Lemma 6 we deduce that for every $x \in \mathscr{H}$ there exists a point $z \in C$ such that

$$
F(z, y)+\left\langle y-g^{-1}(z), g^{-1}(z)-x\right\rangle \geq 0 \quad(y \in C) .
$$

(ii) Let $\left(x, x^{\prime}\right) \in \mathscr{H} \times \mathscr{H}$ and let $z \in J_{F}(x), z^{\prime} \in J_{F}\left(x^{\prime}\right)$. It follows that

$$
\begin{gathered}
F\left(g(z), z^{\prime}\right) \geq\left\langle x-z, z^{\prime}-z\right\rangle, \\
F\left(g\left(z^{\prime}\right), z\right) \geq\left\langle x^{\prime}-z^{\prime}, z-z^{\prime}\right\rangle .
\end{gathered}
$$


Therefore, by using condition (b) from Definition 8 we have that

$$
\begin{aligned}
0 & \geq F\left(g(z), z^{\prime}\right)+F\left(g\left(z^{\prime}\right), z\right) \\
& \geq\left\langle\left(x-x^{\prime}\right)-\left(z-z^{\prime}\right), z^{\prime}-z\right\rangle ;
\end{aligned}
$$

hence we obtain that

$$
\left\langle x-x^{\prime}, z-z^{\prime}\right\rangle \geq\left\|z-z^{\prime}\right\|^{2} .
$$

In particular, for $x=x^{\prime}$, we obtain $z=z^{\prime}$, which implies that $J_{F}$ is single valued. Moreover, from (41) we derive that $J_{F}$ is firmly nonexpansive.

(iii) Let us consider $x \in C$. Then $g(x) \in J_{F}(x)$ means exactly that

$$
F(g(x), y) \geq 0 \quad(y \in C)
$$

hence we obtain the conclusion $g(x) \in S_{F}$.

(iv) It follows from (ii), (iii), and the $g$-equilibrium conditions.

Let us consider the mapping $T_{r}: \mathscr{H} \rightarrow C$ given by

$$
\begin{aligned}
T_{r}(x)=\{z \in C \mid F(z, y) & \\
+\frac{1}{r}\left\langle y-g^{-1}(z), g^{-1}(z)-x\right\rangle \geq 0, & \forall y \in C\} \\
& (r>0) .
\end{aligned}
$$

We are now in position to present an extension of Theorem 3.1 from [2] in the case of our weaker generalized $g$-equilibrium conditions.

Theorem 11. Let $C$ be a nonempty closed convex subset of a Hilbert space $\mathscr{H}$. Let $F: C \times C \rightarrow \mathbb{R}$ a bifunction satisfying the generalized $g$-equilibrium conditions and let $S: C \rightarrow \mathscr{H}$ be a nonexpansive mapping such that

$$
F_{g}(S) \cap E P(f) \neq \emptyset,
$$

where $F_{g}(S)=\{g(v) \in C \mid S(g(v))=v, v \in C\}$. Let $\left(x_{n}\right)_{n \geq 1}$ and $\left(u_{n}\right)_{n \geq 1}$ be two sequences generated by the following recurrent formulas:

$$
x_{1}=x \in \mathscr{H}
$$

$u_{n} \in C$ such that

$$
\begin{gathered}
f\left(u_{n}, y\right)+\frac{1}{r_{n}}\left\langle y-g^{-1}\left(u_{n}\right), g^{-1}\left(u_{n}\right)-x_{n}\right\rangle \geq 0 \\
\quad(y \in C), \\
w_{n}=\left(1-\alpha_{n}\right) x_{n}+\alpha_{n} S\left(u_{n}\right), \\
C_{n}=\left\{z \in H \mid\left\|w_{n}-z\right\| \leq\left\|x_{n}-z\right\|\right\}, \\
D_{n}=\left\{z \in H \mid\left\langle x_{n}-z, x-x_{n}\right\rangle \geq 0\right\}, \\
x_{n+1}=P_{C_{n} \cap D_{n}}(x),
\end{gathered}
$$

for every $n \geq 1$ where $\left(\alpha_{n}\right)_{n \geq 1} \subset[a, 1]$, for some a $\in(0,1)$ and $\left(r_{n}\right)_{n \geq 1} \subset(0, \infty)$ satisfies lim inf ${ }_{n \rightarrow \infty} r_{n}>0$. Then $\left(x_{n}\right)_{n \geq 1}$ converges strongly to $P_{F_{g}(S) \cap E P(f)}(x)$.

Proof. The strategy is based on the model from the proof of Theorem 3.1 in [2]. For the convenience of the reader we present here only the main steps of the proof.

First step consists in proving the fact that the sequence $\left(x_{n}\right)_{n \geq 1}$ is well defined. It is easy to see that $C_{n} \cap D_{n}$ is a closed convex subset of $\mathscr{H}$ for each $n \in \mathbb{N}$. Let $g(v) \in F_{g}(S) \cap \operatorname{EP}(f)$, by using that $T_{r_{n}} x_{n}=u_{n}$ we have that

$$
\left\|u_{n}-g(v)\right\| \leq\left\|T_{r_{n}} x_{n}-T_{r_{n}} v\right\| \leq\left\|x_{n}-v\right\| \quad(n \in \mathbb{N}) .
$$

Moreover, we obtain that

$$
\begin{aligned}
\left\|w_{n}-v\right\| & \leq\left(1-\alpha_{n}\right)\left\|x_{n}-v\right\|+\alpha_{n}\left\|S u_{n}-S g(v)\right\| \\
& \leq\left(1-\alpha_{n}\right)\left\|x_{n}-v\right\|+\alpha_{n}\left\|u_{n}-g(v)\right\| \leq\left\|x_{n}-v\right\| \\
& (n \in \mathbb{N}) .
\end{aligned}
$$

Hence, $v \in C_{n}$ and $F_{g}(S) \cap \operatorname{EP}(f) \subset C_{n}$, for every $n \in \mathbb{N}$. Moreover, by induction we can show that $F(S) \cap \mathrm{EP}(f) \subset C_{n} \cap$ $D_{n}$, for each $n \in \mathbb{N}$. We deduce that $\left(x_{n}\right)_{n \geq 1}$ is well defined, and hence by Lemma 6 the sequence $\left(u_{n}\right)_{n \geq 1}$ is also well defined.

Let us denote by $z^{\prime}=P_{F_{g}(S) \cap \mathrm{EP}(f)}(x)$. Since $x_{n+1}=$ $P_{C_{n} \cap D_{n}}(x)$ we have

$$
\left\|x_{n+1}-x\right\| \leq\left\|z^{\prime}-x\right\| \quad(n \in \mathbb{N}) ;
$$

thus it follows that $\left(x_{n}\right)_{n \geq 1}$ is bounded. Now, by using (47) and (48) we have that $\left(u_{n}\right)_{n \geq 1}$ and $\left(w_{n}\right)_{n \geq 1}$ are also bounded.

Since $x_{n}=P_{D_{n}}(x)$ and $x_{n+1} \in D_{n}$, we have

$$
\left\|x-x_{n}\right\| \leq\left\|x-x_{n+1}\right\| \quad(n \in \mathbb{N}) ;
$$

thus the sequence $C_{n}=\left\|x-x_{n}\right\|, n \geq 1$ is bounded and nondecreasing and there exists $C=\lim _{n \rightarrow \infty}\left\|x-x_{n}\right\|$.

The rest of the proof becomes more similar with the one from Theorem 3.1 in [2], and we recall here only the main steps.

In the first step, it is proved that

$$
\frac{1}{4}\left\|x_{n}-x_{n+1}\right\|^{2} \leq \frac{1}{2}\left\|x-x_{n+1}\right\|^{2}-\frac{1}{2}\left\|x-x_{n}\right\|^{2},
$$

hence $\lim _{n \rightarrow \infty}\left\|x_{n}-x_{n+1}\right\|=0$ and $\lim _{n \rightarrow \infty}\left\|x_{n}-w_{n}\right\|=0$.

By using Lemma 10 we have that

$$
\left\|u_{n}-g(v)\right\|^{2}=\left\|T_{r_{n}}\left(x_{n}\right)-T_{r_{n}}(v)\right\| \leq\left\langle u_{n}-v, x_{n}-v\right\rangle ;
$$

thus we obtain

$$
\left\|u_{n}-g(v)\right\|^{2} \leq\left\|x_{n}-v\right\|^{2}-\left\|x_{n}-v+g(v)-u_{n}\right\|^{2} .
$$

Later on, the fact that $\lim _{n \rightarrow \infty}\left\|x_{n}-v+g(v)-u_{n}\right\|=0$ is proved. Next, we obtain $\lim _{n \rightarrow \infty}\left\|u_{n}-S u_{n}\right\|=0$ and $w \in$ $F_{g}(S) \cap \operatorname{EP}(f)$.

Finally, we conclude that

$$
x_{n} \longrightarrow P_{F_{g}(S) \cap \mathrm{EP}(f)}(x) .
$$




\section{Conflict of Interests}

The author declares that there is no conflict of interests regarding the publication of this paper.

\section{Acknowledgment}

This work was supported by a Grant of the Romanian National Authority for Scientific Research, CNCSUEFISCDI, Project no. PN-II-RU-TE-2011-3-0223.

\section{References}

[1] X. Qin, L.-J. Lin, and S. M. Kang, "On a generalized Ky Fan inequality and asymptotically strict pseudocontractions in the intermediate sense," Journal of Optimization Theory and Applications, vol. 150, no. 3, pp. 553-579, 2011.

[2] A. Tada and W. Takahashi, "Weak and strong convergence theorems for a nonexpansive mapping and an equilibrium problem," Journal of Optimization Theory and Applications, vol. 133, no. 3, pp. 359-370, 2007.

[3] P. L. Combettes and S. A. Hirstoaga, "Equilibrium programming in Hilbert spaces," Journal of Nonlinear and Convex Analysis, vol. 6, no. 1, pp. 117-136, 2005.

[4] S. Takahashi and W. Takahashi, "Strong convergence theorem for a generalized equilibrium problem and a nonexpansive mapping in a Hilbert space," Nonlinear Analysis. Theory, Methods \& Applications, vol. 69, no. 3, pp. 1025-1033, 2008.

[5] M. Bianchi and S. Schaible, "Generalized monotone bifunctions and equilibrium problems," Journal of Optimization Theory and Applications, vol. 90, no. 1, pp. 31-43, 1996.

[6] M. A. Noor and K. I. Noor, “On equilibrium problems," Applied Mathematics E-Notes, vol. 4, pp. 125-132, 2004.

[7] K. Nakajo and W. Takahashi, "Strong convergence theorems for nonexpansive mappings and nonexpansive semigroups," Journal of Mathematical Analysis and Applications, vol. 279, no. 2, pp. 372-379, 2003.

[8] W. Takahashi and M. Toyoda, "Weak convergence theorems for nonexpansive mappings and monotone mappings," Journal of Optimization Theory and Applications, vol. 118, no. 2, pp. 417428, 2003.

[9] C. P. Niculescu and I. Rovenţa, "Fan's inequality in geodesic spaces," Applied Mathematics Letters, vol. 22, no. 10, pp. 15291533, 2009.

[10] C. P. Niculescu and I. Rovenţa, "Fan's inequality in the context of $M_{P}$-convexity," in Applied Analysis and Differential Equations, O. Cârja and I. Vrabie, Eds., pp. 267-274, World Scientific, Singapore, 2007, Proceedings of the International Conference on Applied Analysis and Differential Equations (ICAADE '07).

[11] C. P. Niculescu and I. Rovenţa, "Schauder fixed point theorem in spaces with global nonpositive curvature," Fixed Point Theory and Applications, vol. 2008, Article ID 906727, 8 pages, 2009.

[12] J. M. Borwein and A. S. Lewis, Convex Analysis and Nonlinear Optimization. Theory and Examples, Springer, New York, NY, USA, 2000. 


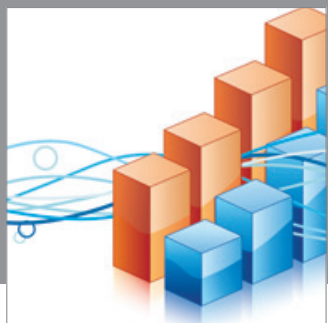

Advances in

Operations Research

mansans

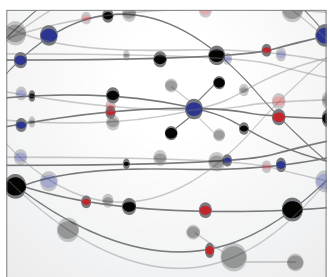

The Scientific World Journal
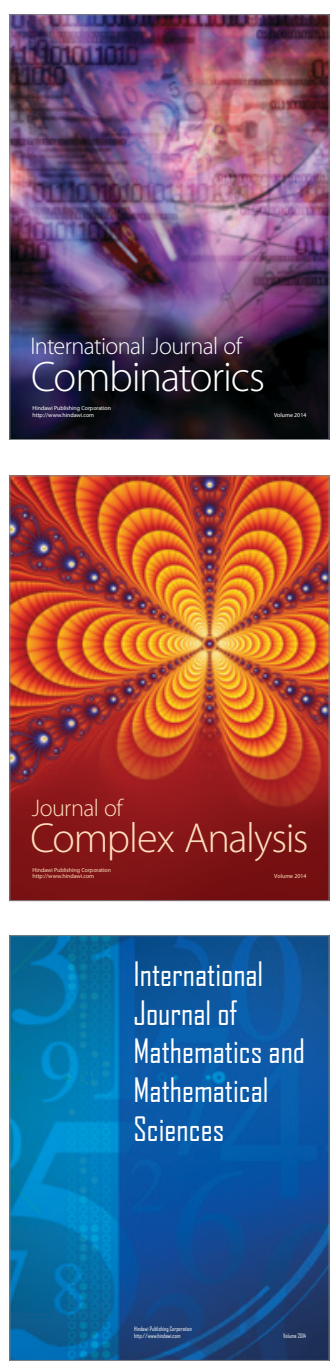
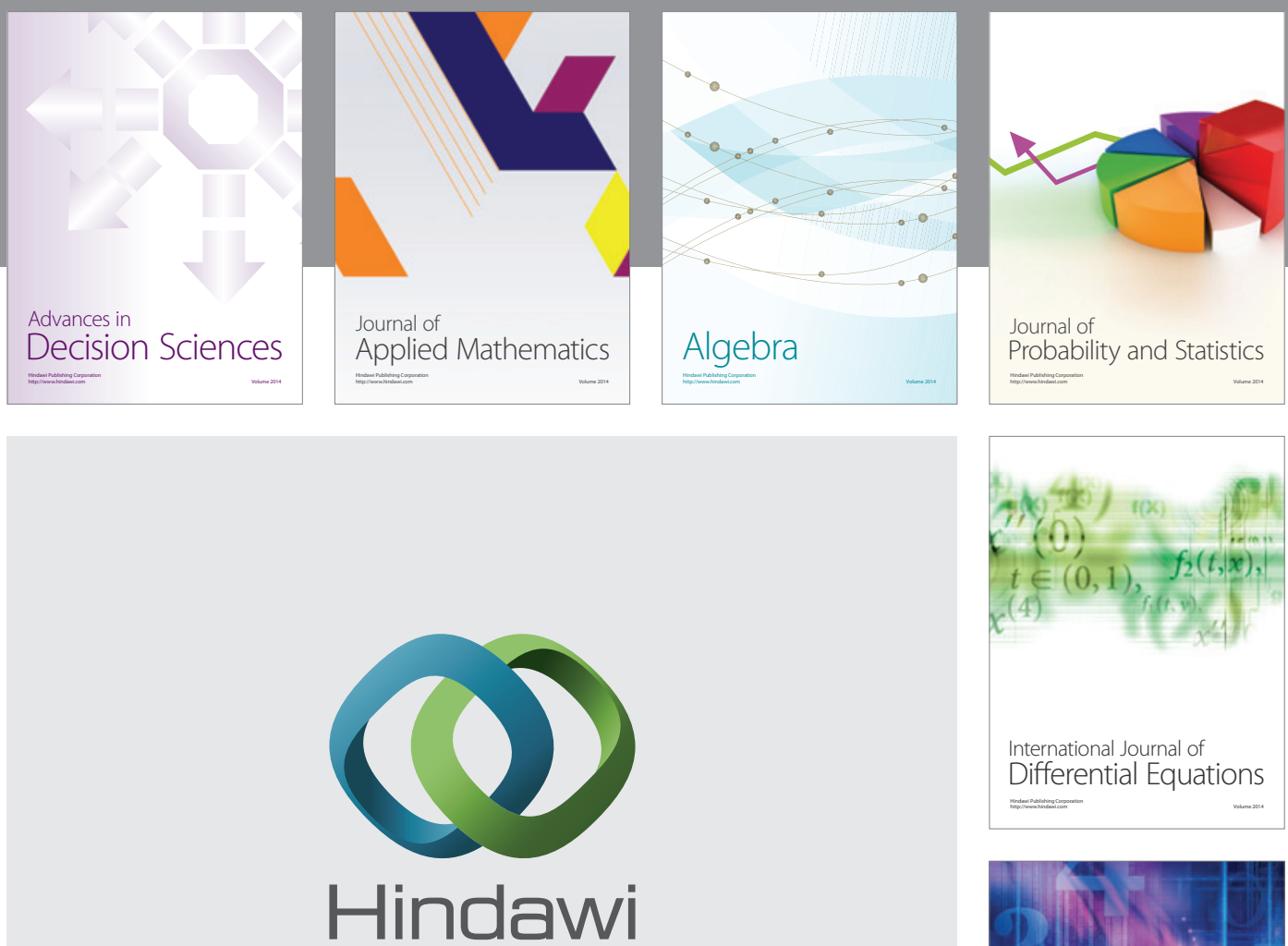

Submit your manuscripts at http://www.hindawi.com
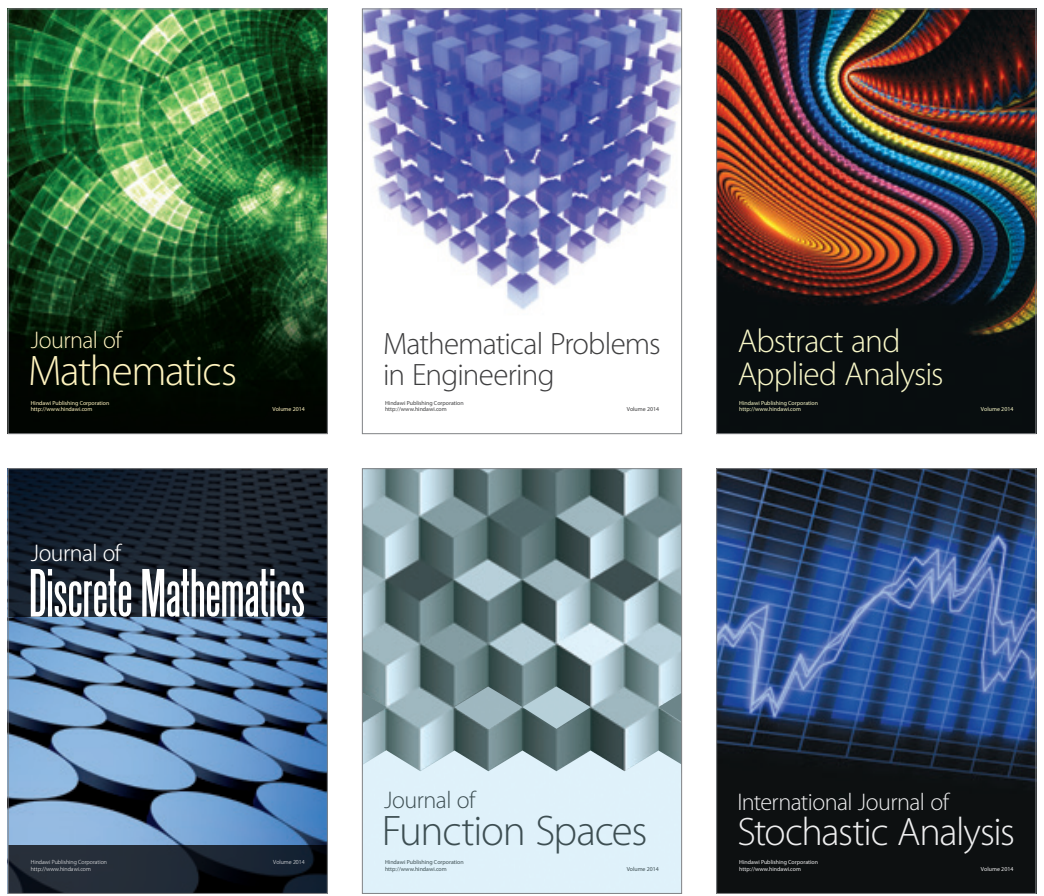

Journal of

Function Spaces

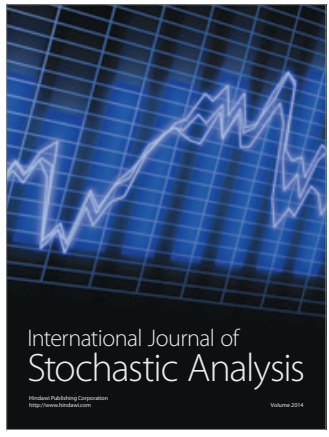

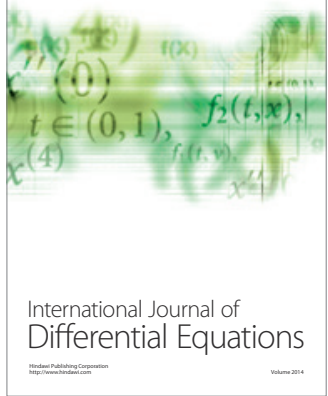
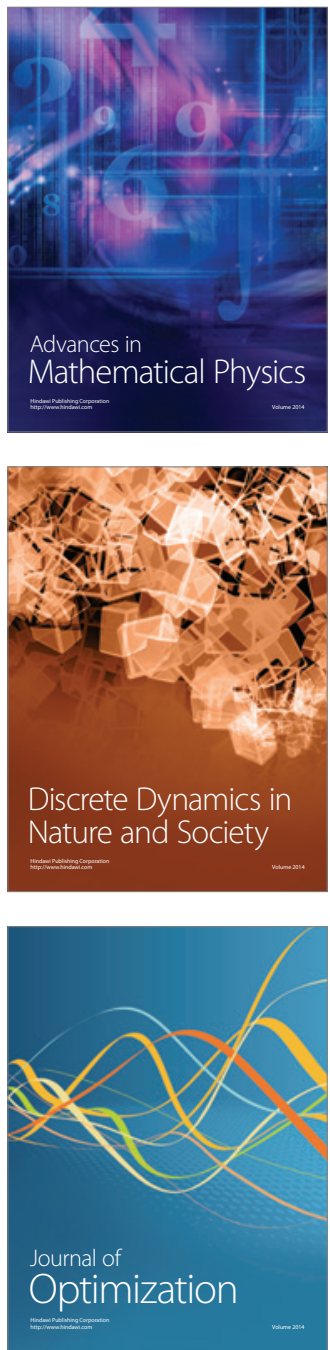